\title{
MICROVASCULAR RESEARCH
}

Accepted January $1^{\text {st }} 2018$

ISSN: 0026-2862; IMPACT FACTOR= 2.574

Publisher: Elsevier (USA)

Editor-in-Chief: Prof. Dr. P.A. D'Amore, Harvard Medical School, Boston, Massachusetts, USA.

\author{
STUDY OF MICROVASCULAR BLOOD FLOW MODULATED BY ELECTROOSMOSIS \\ ${ }^{* 1}$ Dharmendra Tripathi, ${ }^{1}$ Ashu Yadav, ${ }^{2} \mathrm{O}$. Anwar Bég and ${ }^{1}$ Rakesh Kumar \\ ${ }^{I}$ Department of Mechanical Engineering, Manipal University Jaipur, Rajasthan-303007, India. \\ ${ }^{2}$ Fluid Mechanics and Propulsion, Aeronautical and Mechanical Engineering, University of Salford, \\ Newton Building, G77, The Crescent, Salford, M54WT, England, UK. \\ *Corresponding author-email: dharmendra.tripathi@jaipur.manipal.edu
}

\begin{abstract}
An analytical study of microvascular non-Newtonian blood flow is conducted incorporating the electro-osmosis phenomenon. Blood is considered as a Bingham rheological aqueous ionic solution. An externally applied static axial electrical field is imposed on the system. The Poisson-Boltzmann equation for electrical potential distribution is implemented to accommodate the electrical double layer (EDL) in the microvascular regime. With long wavelength, lubrication and Debye-Hückel approximations, the boundary value problem is rendered non-dimensional. Analytical solutions are derived for the axial velocity, volumetric flow rate, pressure gradient, volumetric flow rate, averaged volumetric flow rate along one time-period, pressure rise along one wavelength and stream function. A plug width is featured in the solutions. Via symbolic software (Mathematica ${ }^{\mathbf{T M}}$ ), graphical plots are generated for the influence of Bingham plug flow width parameter, electrical Debye length (thickness) and Helmholtz-Smoluchowski velocity (maximum electro-osmotic velocity) on the key hydrodynamic variables. An increase in plug flow width is observed to accelerate the axial flow, enhance volumetric flow rate and has a varied influence on the pressure rise depending on whether the flow is in the free pumping or pumping region. Increasing electrical Debye length consistently enhances axial flow, volumetric flow rate and also pressure rise (at any value of volumetric flow rate).
\end{abstract}

KEYWORDS: Electroosmosis; Bingham plastic fluids; electric double layer; trapping; Blood flow; plug flow. 


\section{INTRODUCTION}

Electrokinetic transport has emerged as a vibrant area of modern fluid mechanics. It involves both analysis and experimental investigations of the interaction of electrolytic fluids and external electric fields which may be static or alternating. It features many complex and intriguing phenomena including electric double layers, wetted surfaces, zeta potentials and charge distributions. Electrokinetics includes electro-osmosis, electrophoresis, diffusiophoresis (where chemical gradients are significant) and various other phenomena. In recent years electro-osmotic (EO) flows in particular have penetrated many different microand nanoscale technological applications including nanoscale electro-fluid thruster designs in space propulsion [1], non-adsorbing polymer injection systems [2], ion-exchange membrane designs [3], biochip fabrication [4], micro-channels [5], microbial fuel cells in carbon capture [6], dewatering in fossil fuel energy processes [7], corrosion mitigation in civil engineering [8], nano-bot propulsion for medical treatment [9] and novel pump designs for microfluidic chromatography. Other applications include laser diode arrays cooling and biological microreactors. Electro-osmotic driven flow also achieves optimized flow profiles and is very beneficial in conveying sensitive samples. Electroosmotic pumping in micro-channels is characterized by a low Reynolds number and therefore inertial forces are dominated essentially by viscous hydrodynamic forces. At small scales the impact of electrical forces is significantly improved in electroosmotics. In parallel with clinical and industrial synthesis of electroosmotic devices, mathematical modelling of electroosmotic phenomena has also intensified in recent years since simulation provides an important compliment to experimental works. Soong et al. [11] analysed numerically the electrokinetic thermal-fluid flow in electroosmotic transport in a rotating dual rotor-stator disk system as a model of microfluidics, solving the transformed Navier-Stokes equations coupled with the Poisson electrical potential and Nernst-Plank convection-diffusion ionic equations. Bianchi et al. [12] considered the electroosmotic flow in complex geometries using a finite element code. Wang et al. [13] used a lattice PoissonBoltzmann method (LPBM) to investigate the dynamics of electroosmotic transport in anisotropic permeable materials containing ellipse arrays packed in a microchannel. These studies assumed the electrolytic solution to be Newtonian. However many working fluids in microfluidic systems exhibit non-Newtonian characteristics which dramatically alter flow characteristics. Non-Newtonian electroosmotic fluid mechanics has provided a rich arena for theoretical and computational studies and has stimulated the application of many different rheological material models. Ng [14] employed the Ostwald-de Waele power-law model to investigate electroosmotic rheological pumping in a slit micro-channel with variable geometric 
and zeta potential characteristics, considering both pseudoplastic and dilatant cases. Cho et al. [15] also used the power-law model to analyse the mixing flow in a contracting-expanding microchannel noting that mixing efficiency is decreased with decreasing rheological flow behaviour index. Huang et al. [16] also employed the power-law model to confirm experimentally (via electrical current monitoring and microscopy fluorescence methods) that electroosmotic driven flow is enhanced with shear-thinning behaviour in rectangular microchannels. Jiménez et al. [17] used the Maxwell viscoelastic model to simulate the electroflow of ionic solutions in rectangular microchannels with large asymmetric wall zeta potentials, addressing both relaxation time effect and noting that velocity assumes a transient oscillatory trend caused by the competition between viscous, elastic and electroosmotic forces. Kaushik et al. [18] implemented the Oldroyd-B viscoelastic model for unsteady rotational electroosmotic flow in a microfluidic channel, motivated by hemodynamic microfluidic systems. Ng [19] utilized a viscoplastic Casson model for steady electroosmotic (EO) flow in a parallel-plate microchannel, noting that the yield surface delineates the regime into sheared and unsheared zones and that the opposing effect of yield stress on volumetric flow rate may be compensated for via pressure gradient. Further studies of electroosmotic rheological hydrodynamics have deployed thixotropic models [20] and the viscoelastic Phan-ThienTanner model [21]. A further important rheological model is the Bingham viscoplastic model. This simulates quite accurately actual biological fluids for which a yield stress, a critical value of stress must be attained before flow is initiated. Originally introduced to simulate paints, owing to its simple and robust formulation, the Bingham model has been utilized in many diverse areas of engineering sciences including petroleum slurries [22], mud flows in coastal engineering [23], and microscale thermal engineering systems [24, 25].

In the above studies the conduits considered e.g. micro-channels, have been assumed to possess rigid i.e. non-flexible boundaries (walls). With the recent upsurge in biomimetic and biologically-inspired designs, flexible boundaries have become attractive. An important biophysical mechanism which exploits wall deformability is peristalsis. In many physiological processes, as elaborated by Fung [26], bio-fluids are trans-located transportation by rhythmic muscles contraction followed by relaxation. This highly efficient and adaptive mechanism of internal fluid propulsion arises in embryology, intestinal pumping, vaso-motion in small blood vessels, swallowing, lymph dynamics, water transport in botany etc. A classical investigation of the pumping characteristics in peristalsis was presented many decades ago by Shapiro et al. [27] who established much of the methodology for low Reynolds number long wavelength 
models and popularized the transformation from the wave (i.e. moving) frame and the laboratory (fixed) frame. They derived relationships for the free pumping, augmented pumping and pumping regions and also analysed for the first time carefully the tapping and reflux phenomena in two-dimensional situations. More recently Walker and Shelley [28] presented a generalized variational method for optimizing the wave shape in two-dimensional peristaltic pumping in a channel using a sequential quadratic programming (SQP) method and mimicking wall deformation via an explicit front-tracking approach over a wide spectrum of fluxes and Reynolds numbers. Two-dimensional peristaltic flows containing suspended particles were earlier studied using asymptotic methods by Kaimal [29]. Tang and Shen [30] used Stokes and long wave asymptotic expansions to simulate the peristaltic pumping of a heat-conducting fluid in cylindrical vessels with a prescribed pressure drop, and Newton's cooling law enforced at the vessel wall. These studies were however confined to Newtonian viscous flows. An extensive range of micro-scale devices utilize non-Newtonian working fluids. Peristaltic micro-channel rheological flows provide a rich arena for simulation. Nagarani et al. [31] used the Casson viscoplastic model to examine the annular pumping of a polymer under peristaltic waves, observing that both yield stress and annular gap dramatically modify the pressure rise, frictional wall resistance and synthesis and evolution of trapped boluses. Further nonNewtonian peristaltic hydrodynamic studies include Tripathi and Bég [32] who deployed a fractional second-grade viscoelastic model for oblique peristaltic pumping and Abd elmaboud and Mekheimer [33] who examined peristaltic transport in porous media-filled conduits with the Reiner-Rivlin differential second order viscoelastic model. Several studies of peristaltic pumping with the Bingham plastic model have also been communicated in recent years. Khabazi et al. [34] used the bi-viscous (Bingham) model and the multiple-relaxation-time lattice Boltzmann method (MRT-LBM) to compute peristaltic pumping characteristics in a planar two-dimensional channel, noting that for yield-stress fluids there arises a threshold wave number above which the yield stress accelerates axial flow and that yield stress causes a contraction in bolus size. Tripathi and Bég [35] explored a variety of viscoplastic models for peristaltic pumping including the Vocadlo model, Bingham model and Casson model. Electroosmotic Newtonian and non-Newtonian peristaltic flows have also attracted some attention in recent years. Chakraborty [36] considered power-law electrolytes in micro-channel peristaltic transport. Tripathi et al. [37] studied Debye length effects on electroosmotic Newtonian pumping in capillary blood flows. Goswami et al. [38] used both Newtonian (for the wall adhering layer) and non-Newtonian (for the core region) models to investigate electroosmotic wavy flow in microvascular hemodynamics. Further analyses include Tripathi 
et al. [39] who used the Jefferys viscoelastic model, Shit et al. [40] who employed a powerlaw model and also considered heat transfer and Joule dissipation effects and very recently Tripathi et al. [41] who employed Stokes' couple stress rheological model.

To the authors' knowledge, thus far, the electroosmotic peristaltic pumping of Bingham plastic fluids has not been scrutinized for the case of complex wavy micro-channels i.e. where different amplitude waves propagate along both upper and lower micro-channel walls. However the system does retain symmetry about the micro-channel centre line. The present study addresses this configuration for the first time. Detailed analytical solutions for the transformed, non-dimensional boundary value problem are presented. Extensive interpretation of the impact of electroosmotic parameters and viscoelasticity (yield stress) on peristaltic flow characteristics are evaluated. Bolus dynamics is also visualized. The present closed-form solutions are numerically evaluated with physically relevant data using Mathematica symbolic software. The solutions present an important benchmark also for more general microvascular blood flow models and experimental studies.

\section{ELECTRO-OSMOTIC PERISTALTIC VISCOPLASTIC MODEL}

\subsection{Flow regime}

We consider electroosmotic flow of Bingham viscoplastic fluids through a complex wavy twodimensional microchannel. The geometry of the channel is illustrated in Fig.1 and wall deformation is mathematically expressed as:

$\bar{h}(\bar{x}, \bar{t})=a+\sum_{i=1}^{m} \bar{\phi}_{i} \sin \frac{2 i \pi}{\lambda}(\bar{x}-c \bar{t})$,

where $\bar{h}, \bar{x}, \bar{t}, a, \bar{\phi}_{i}, \lambda$ and $c$ represent transverse vibration of the wall, axial coordinate, time, half width of the channel, amplitude of the different $(m)$ waves, wavelength and wave velocity respectively. Furthermore, $a$ and $\bar{\phi}_{i}$ satisfy the condition: $a \leq \sum_{i=1}^{n} \bar{\phi}_{i}$.

\subsection{Bingham Plastic Fluid Model}

The constitutive equations for a Bingham viscoplastic fluid is expressed as:

$$
\left.\begin{array}{l}
\bar{\tau}=\bar{\tau}_{\mathrm{o}}+\mu \dot{\gamma}, \bar{\tau} \geq \bar{\tau}_{\mathrm{o}} \\
\dot{\gamma}=0, \quad \bar{\tau}<\bar{\tau}_{\mathrm{o}}
\end{array}\right\}
$$


where $\bar{\tau}$ is the shear stress, $\bar{\tau}_{0}$ is the yield stress, $\dot{\gamma}$ is the rate of shear strain, $\mu$ is the consistency of viscoplastic material.

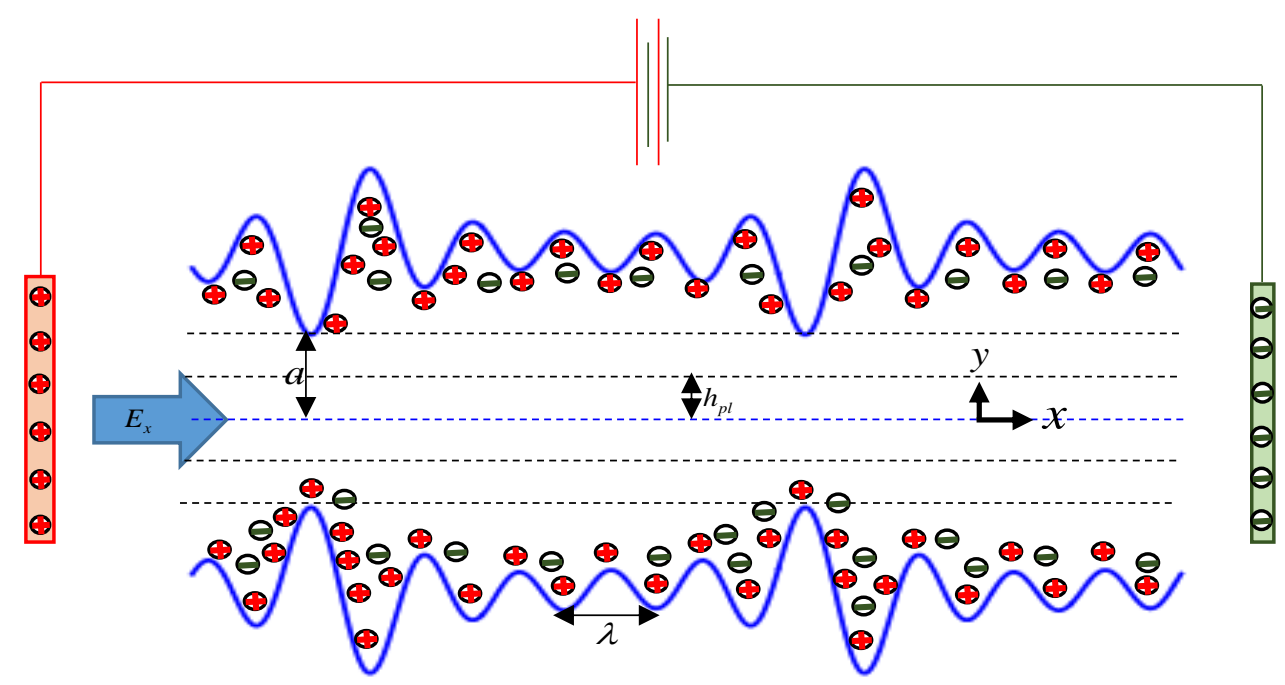

Fig.1. Geometrical representation of electroosmotic flow through complex wavy microchannel.

\subsection{Governing Equations}

In this model, we consider the electroosmotic flow of non-Newtonian aqueous ionic solution altered by means of an externally applied electric field along the length of channel. The governing equations are considered as:

$\frac{\partial \bar{u}}{\partial \bar{x}}+\frac{\partial \bar{v}}{\partial \bar{y}}=0$

$\rho\left(\frac{\partial}{\partial \bar{t}}+\bar{u} \frac{\partial}{\partial \bar{x}}+\bar{v} \frac{\partial}{\partial \bar{y}}\right) \bar{u}=-\frac{\partial p}{\partial \bar{x}}+\frac{\partial \bar{\tau} \overline{x x}}{\partial \bar{x}}+\frac{\partial \bar{\tau} \overline{x y}}{\partial \bar{y}}+\rho_{e} E_{x}$,

$\rho\left(\frac{\partial}{\partial \bar{t}}+\bar{u} \frac{\partial}{\partial \bar{x}}+\bar{v} \frac{\partial}{\partial \bar{y}}\right) \bar{v}=-\frac{\partial \bar{p}}{\partial \bar{y}}+\frac{\partial \bar{\tau}_{\overline{y x}}}{\partial \bar{x}}+\frac{\partial \bar{\tau}_{\overline{y y}}}{\partial \bar{y}}+\rho_{e} E_{y}$

where, $\bar{\tau}_{\overline{x x}}, \bar{\tau}_{\overline{x y}}, \bar{\tau}_{\overline{y x}}, \bar{\tau}_{\overline{y y}}$ are the extra stress components and $\rho, \bar{u}, \bar{v}, \bar{p}, \mu$, and $E_{x} \& E_{y}$ denote the fluid density, axial velocity, transverse velocity, pressure, fluid dynamic viscosity, and electrical field in axial and transverse directions, respectively.

The positive ions $\bar{n}_{+}$and negative ion $\bar{n}_{-}$are both assumed to have bulk concentration (number density) $\bar{n}_{0}$, and valencies of $\bar{z}_{+}$and $\bar{z}_{-}$respectively. For simplicity, we consider the electrolyte 
to be a $\bar{z}: \bar{z}$ symmetric electrolyte, i.e. $\bar{z}_{+}=-\bar{z}_{-}=\bar{z}$. The charge number density is related to the electrical potential in the transverse direction $(\bar{\varphi})$ through the Poisson equation:

$$
\nabla^{2} \bar{\varphi}=-\frac{\rho_{e}}{\varepsilon},
$$

where $\varepsilon$ is the electrical permittivity of the ionic solution. Further, in order to determine the potential distribution, we need to describe the charge number density. For this, the ionic number distributions of the individual species are given by the Nernst-Planck equation for each species as:

$$
\frac{\partial \bar{n}_{ \pm}}{\partial \bar{t}}+\bar{u} \frac{\partial \bar{n}_{ \pm}}{\partial \bar{x}}+\bar{v} \frac{\partial \bar{n}_{ \pm}}{\partial \bar{y}}=D\left(\frac{\partial^{2} \bar{n}_{ \pm}}{\partial \bar{x}^{2}}+\frac{\partial^{2} \bar{n}_{ \pm}}{\partial \bar{y}^{2}}\right) \pm \frac{D z e}{k_{B} T}\left(\frac{\partial}{\partial \bar{x}}\left(\bar{n}_{ \pm} \frac{\partial \bar{\varphi}}{\partial \bar{x}}\right)+\frac{\partial}{\partial \bar{y}}\left(\bar{n}_{ \pm} \frac{\partial \bar{\varphi}}{\partial \bar{y}}\right)\right),
$$

Here it is assumed that there are equal ionic diffusion coefficients for both the species, and that the mobility of the species is given by the Einstein formula where $D$ represents the diffusivity of the chemical species, $T$ is the average temperature of the electrolytic solution and $k_{B}$ is Boltzmann constant.

To facilitate analytical solutions of equations (2)-(7), it is advantageous to introduce a group of non-dimensional parameters; $x=\frac{\bar{x}}{\lambda}, y=\frac{\bar{y}}{a}, t=\frac{\overline{t c}}{\lambda}, u=\frac{\bar{u}}{c}, v=\frac{\bar{v}}{\delta c}, h=\frac{\bar{h}}{a}, \phi_{i}=\frac{\bar{\phi}_{i}}{a}, \delta=\frac{a}{\lambda}$, $\tau=\frac{\bar{\tau} a}{\mu c}, \tau_{0}=\frac{\overline{\tau_{0}} a}{\mu c}, p=\frac{\bar{p} a^{2}}{\mu c \lambda}, \varphi=\frac{z e \bar{\varphi}}{k_{B} T}, n=\frac{\bar{n}}{n_{0}}$, where, $x$ and $y$ are dimensionless longitudinal (axial) and transverse coordinates respectively, $t$ is non-dimensional time, $h$ is dimensionless transverse vibration of the wall, dimensionless amplitude of the different $(m)$ waves, $\delta$ is peristaltic wave number, $\tau$ is dimensionless shear stress, $\tau_{0}$ is the non-dimensional yield stress, $p$ is non-dimensional pressure, $\varphi$ is normalized electrical potential and $\mathrm{n}$ is dimensionless number density of the Bingham electrolyte. The nonlinear terms in the Nernst Planck equations are $O\left(P e \delta^{2}\right)$, where $P e$ represents the ionic Peclét number $(P e=\operatorname{Re} S c), S c=\mu / \rho D$ denotes the Schmidt number and $\operatorname{Re}=\frac{\rho c \lambda}{\mu}$ denotes the Reynolds number based on peristaltic wave length. The nonlinear terms in the momentum equation are found to be $O\left(\operatorname{Re} \delta^{2}\right)$. Therefore, the nonlinear terms may be dropped in the limit that $\operatorname{Re}, P e, \delta<<1$. In the above approximations, the emerging Poisson equation is: 
$\frac{\partial^{2} \varphi}{\partial y^{2}}=-\kappa^{2}\left(\frac{n_{+}-n_{-}}{2}\right)$

where $\kappa=a e z \sqrt{\frac{2 n_{0}}{\varepsilon K_{B} T}}$, is inverse of Debye length (or EDL thickness). Additionally the ionic distribution may be determined by means of the simplified Nernst Planck equations:

$0=\frac{\partial^{2} n_{ \pm}}{\partial y^{2}} \pm \frac{\partial}{\partial y}\left(n_{ \pm} \frac{\partial \varphi}{\partial y}\right)$

Eqns (9) are subjected to $n_{ \pm}=1$ at $\varphi=0$ and $\partial n_{ \pm} / \partial y=0$ where $\partial \varphi / \partial y=0$ (bulk conditions). These yield the much-celebrated Boltzmann distribution for the ions:

$n_{ \pm}=e^{\mp \varphi}$

Combining equation (8) and (10), we obtain the Poisson-Boltzmann paradigm for the potential determining the electrical potential distribution;

$\frac{\partial^{2} \varphi}{\partial \bar{y}^{2}}=\kappa^{2} \sinh (\varphi)$

In order to make further analytical progress, equation (11) requires simplification. Equation (11) may be linearized under the low-zeta potential approximation. This assumption is not ad hoc since for a wide range of $\mathrm{pH}$ values, the magnitude of zeta potential is less than $25 \mathrm{mV}$. Therefore, equation (11) may be contracted to:

$\frac{\partial^{2} \varphi}{\partial y^{2}}=\kappa^{2} \varphi$

which may be solved subjected to $\left.\frac{\partial \varphi}{\partial y}\right|_{y=0}=0$ and $\left.\varphi\right|_{y=h}=1$. The resulting potential function is obtained as: 
$\varphi=\frac{\cosh (\kappa y)}{\cosh (\kappa h)}$

After employing the large wavelength and low Reynolds number approximation, the Eqs. (1-

5) are reduced to:

$\left.\begin{array}{l}h(x, t)=1+\sum_{i=1}^{m} \phi_{i} \sin 2 i \pi(x-t), \\ \tau_{x y}=\tau_{0}+\left(\frac{\partial u}{\partial y}\right), \tau_{x y}>\tau_{0} \\ \frac{\partial u}{\partial y}=0, \quad \tau>\tau_{0}\end{array}\right\}$

$\frac{\partial u}{\partial x}+\frac{\partial v}{\partial y}=0$

$\frac{\partial \tau_{x y}}{\partial y}=\frac{\partial p}{\partial x}-\kappa^{2} u_{e} \frac{\cosh (\kappa y)}{\cosh (\kappa h)}$,

$\frac{\partial p}{\partial y}=0$

where, $u_{e}=-\frac{E_{x} \varepsilon \zeta}{\mu c}$ is the Helmholtz-Smoluchowski velocity. The associated normalized boundary conditions are:

$\left.\tau_{x y}\right|_{y=0}=0,\left.\tau_{x y}\right|_{y=h_{p l}}=\tau_{0},\left.u\right|_{y=h}=0$,

where, $h_{p l}$, is width of the plug flow region.

\subsection{Analytical Solution}

Using Eq.(15) and boundary condition (19), the axial velocity from Eq.(17) is obtained as:

$u=\frac{1}{2} \frac{\partial p}{\partial x}\left(y^{2}-h^{2}\right)-h_{p l} \frac{\partial p}{\partial x}(y-h)+u_{e}\left(1-\frac{\cosh (\kappa y)}{\cosh (\kappa h)}+\kappa(-h+y) \frac{\sinh \left(h_{p l} \kappa\right)}{\cosh (\kappa h)}\right), h_{p l}<y<h$

The volumetric flow rate in $h_{p l}<y<h$ is defined as follows:

$Q=\int_{h_{p l}}^{h} u d y$.

After integration, the appropriate expression is:

$Q=\frac{1}{3}\left(h-h_{p l}\right)^{3} \frac{\partial p}{\partial x}-\left(-h+h_{p l}\right) u_{e}-\frac{u_{e}}{\kappa}\left(-1+\frac{1}{2}\left(h-h_{p l}\right)^{2} \kappa^{2}\right) \frac{\sinh \left(h_{p l} \kappa\right)}{\cosh (\kappa h)}-\frac{u_{e}}{\kappa} \tanh (h \kappa)$. 
Manipulating Eq.(22), the pressure gradient is derived as:

$\frac{\partial p}{\partial x}=\frac{3}{\left(h-h_{p l}\right)^{3}}\left\{Q+u_{e}\left(\left(-h+h_{p l}\right)+\frac{1}{\kappa}\left(-1+\frac{1}{2}\left(h-h_{p l}\right)^{2} \kappa^{2}\right) \frac{\sinh \left(h_{p l} \kappa\right)}{\cosh (\kappa h)}+\frac{1}{\kappa} \tanh (h \kappa)\right)\right\}$.

The pressure rise across the one wavelength can be expressed as:

$\Delta p=\int_{0}^{1} \frac{\partial p}{\partial x} d x$

The transformations between the wave frame $\left(x_{w}, y_{w}\right)$ moving with velocity $(c)$ and the fixed (laboratory) frame $(x, y)$ are given by:

$x=x_{w}-c t, \quad y=y_{w}, \quad u=u_{w}+c, \quad v=v_{w}$,

where $\left(u_{w}, v_{w}\right)$ and $(u, v)$ are the velocity components in the wave and fixed frame respectively.

The volumetric flow rate in the wave frame is given by

$q_{w}=\int_{0}^{h} u_{w} d y_{w}=\int_{0}^{h}(u-1) d y_{w}$,

On integration this yields:

$q_{w}=Q-h$.

Averaging volumetric flow rate along one time period, we get:

$\bar{Q}=\int_{0}^{1} Q d t=\int_{0}^{1}\left(q_{w}+h\right) d t$,

which, on integration, yields

$\bar{Q}=q_{w}+1=Q+1-h$.

The stream function is defined as: $u_{w}=\frac{\partial \psi}{\partial y_{w}}, v_{w}=-\frac{\partial \psi}{\partial x_{w}}$, which yields:

$$
\begin{aligned}
\psi= & -\frac{1}{4\left(h-h_{p l}\right)^{3} \kappa \cosh (h \kappa)}\left(2 \kappa y \left(h^{3} u_{e}+2 h_{p l}^{3} u_{e}-3 h^{2}\left(Q+h_{p l} u_{e}\right)-3 h_{p l}{ }^{2} u_{e} y+Q y^{2}+h_{p l} y\left(-3 Q+u_{e} y\right)\right.\right. \\
& \left.+h\left(6 h_{p l} Q+3 h_{p l} u_{e} y-u_{e} y^{2}\right)\right) \cosh (h \kappa)+u_{e}\left(2 y\left(-3 h^{2}+6 h h_{p l}+y\left(-3 h_{p l}+y\right)\right) \sinh (h \kappa)\right. \\
& +y\left(h^{4} \kappa^{2}-2 h^{3} \kappa^{2} y+y\left(6 h_{p l}-h_{p l}^{3} \kappa^{2}-2 y+h_{p l}^{2} \kappa^{2} y\right)+2 h h_{p l}\left(-6+h_{p l}^{2} \kappa^{2}-\kappa^{2} y^{2}\right)\right. \\
& \left.\left.\left.+h^{2}\left(6-3 h_{p l}^{2} \kappa^{2}+3 h_{p l} \kappa^{2} y+\kappa^{2} y^{2}\right)\right) \sinh \left(h_{p l} \kappa\right)+4\left(h-h_{p l}\right)^{3} \sinh (\kappa y)\right)\right) .
\end{aligned}
$$

\section{COMPUTATIONAL RESULTS AND DISCUSSION}

Via Mathematica symbolic software, numerical evaluation of the closed-for solutions is conducted for selected parameters. The results are plotted in figs. 2- 13. 


\subsection{Effects of yield stress}

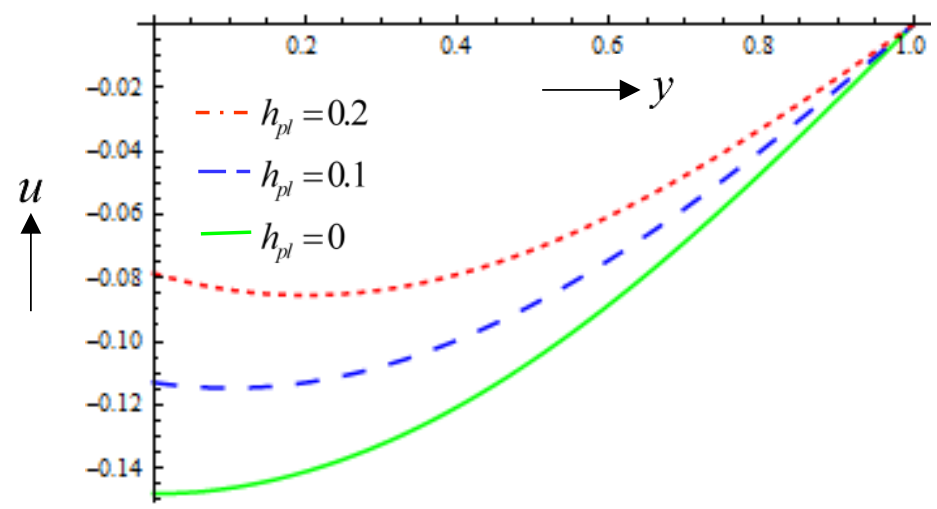

Fig.2. Effect of plug flow width on axial velocity profile with $x=1, \kappa=1, u_{e}=1$.

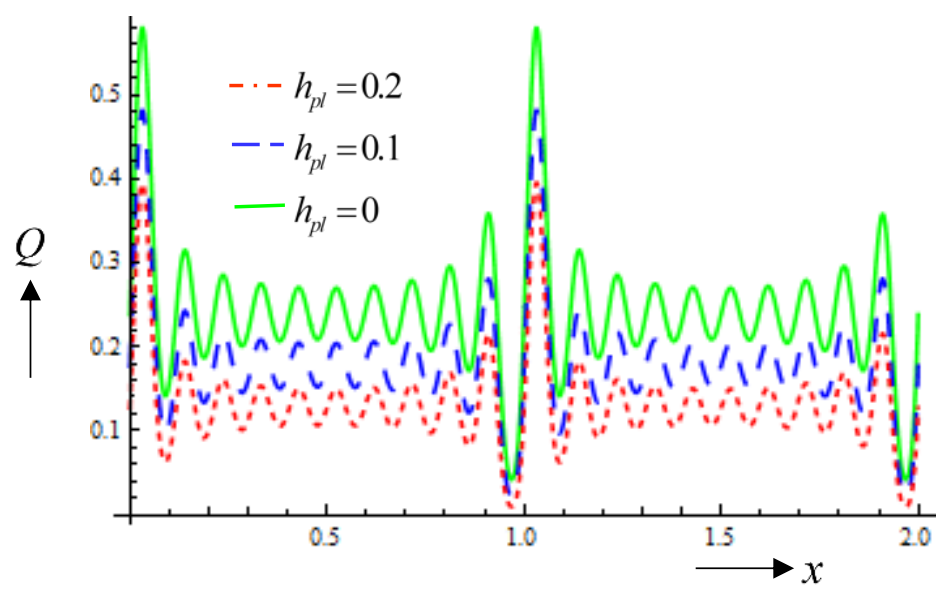

Fig.3. Effect of plug flow width on volumetric flow rate with $\kappa=1, u_{e}=1$.

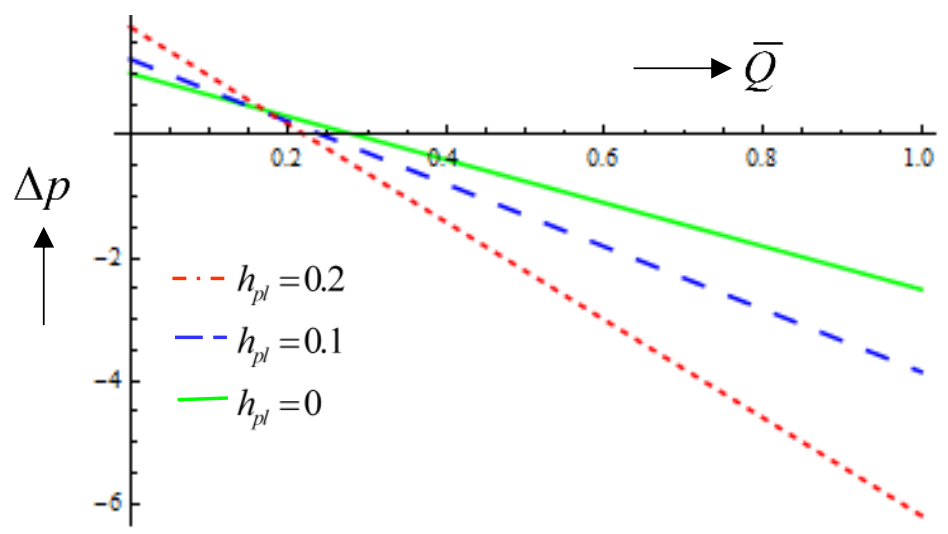

Fig.4. Effect of plug flow width on pressure rise with $\kappa=1, u_{e}=1$. 

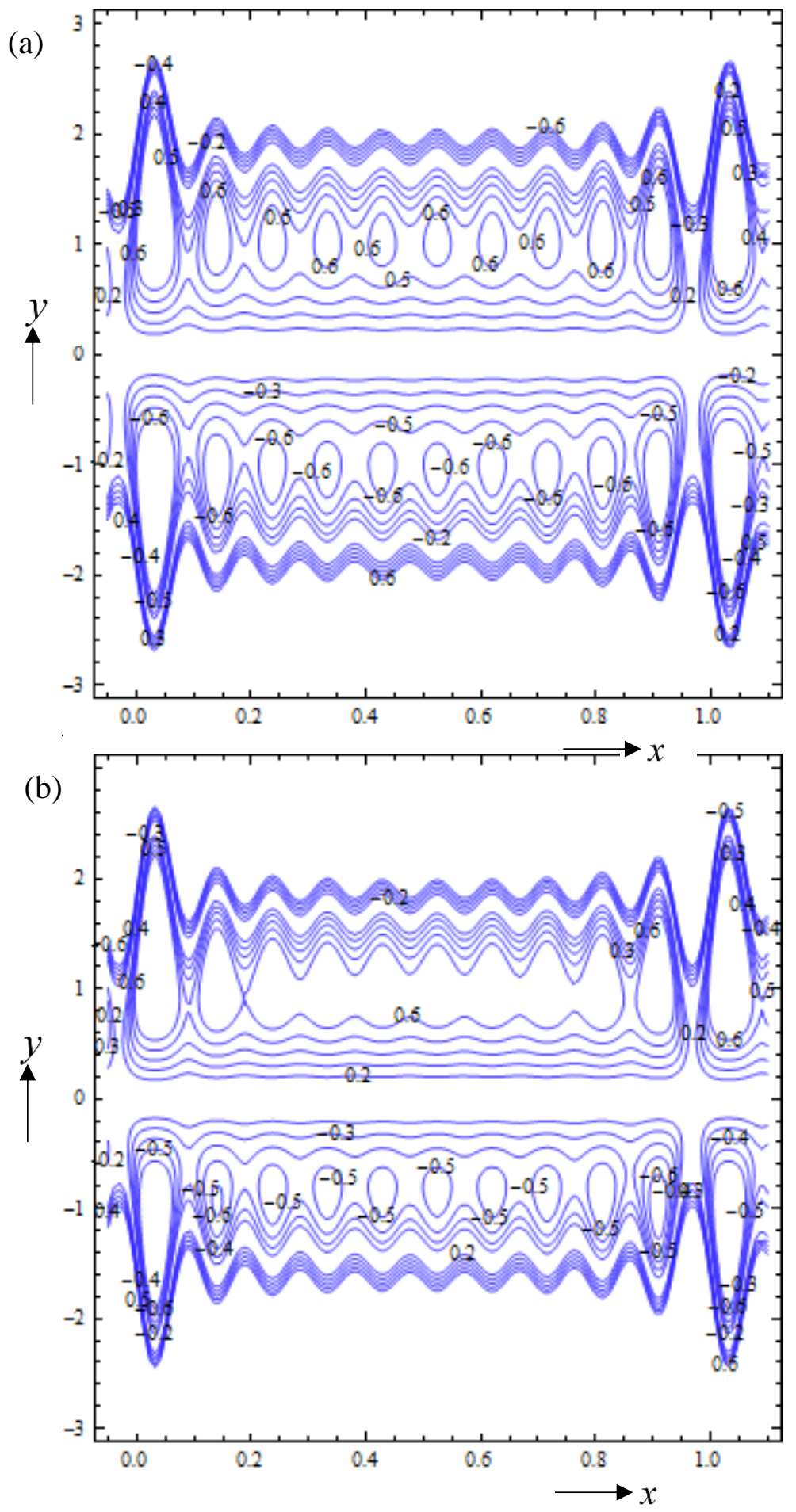


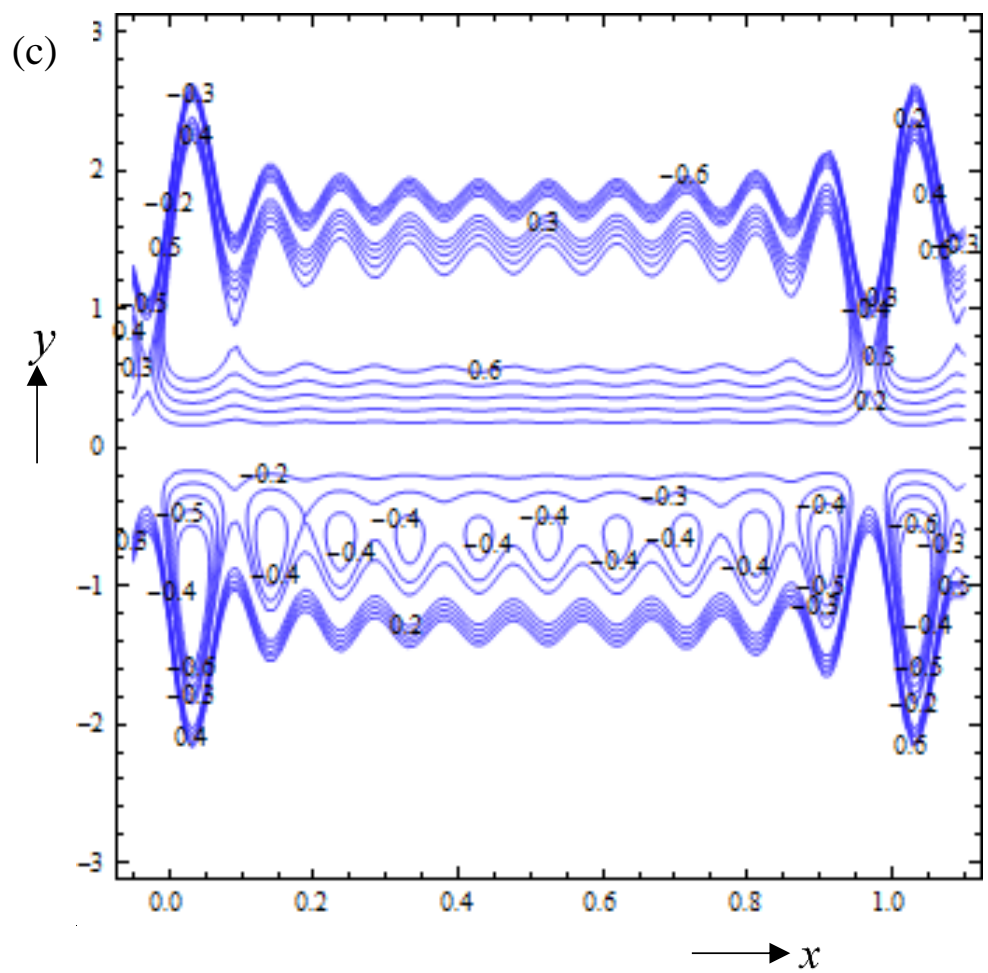

Fig.5. Stream lines for $\bar{Q}=0.6, \kappa=1, u_{e}=1$ with (a) $h_{p l}=0$ (b) $h_{p l}=0.1$ (c) $h_{p l}=0.2$

\subsection{Effects of EDL thickness}

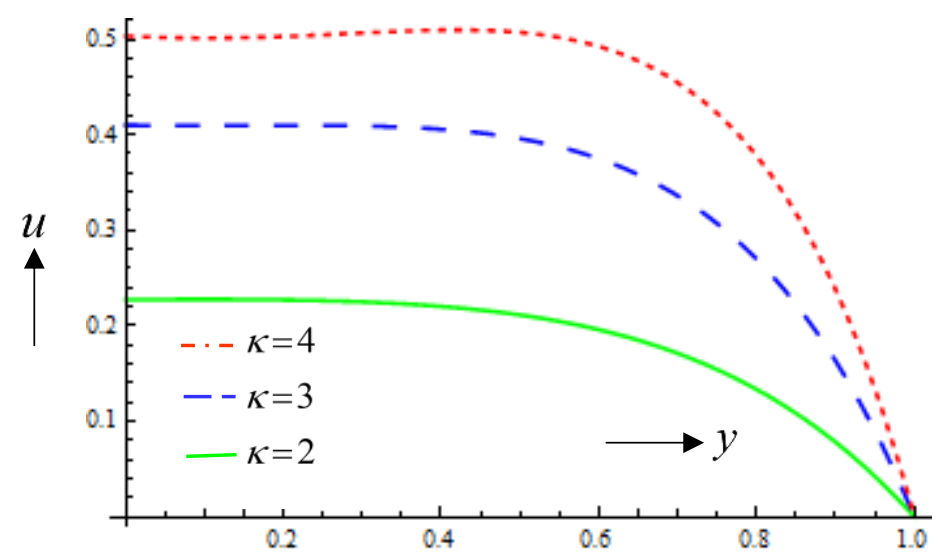

Fig.6. Effect of Debye length on axial velocity with $x=1, h_{p l}=0.1, u_{e}=1$. 


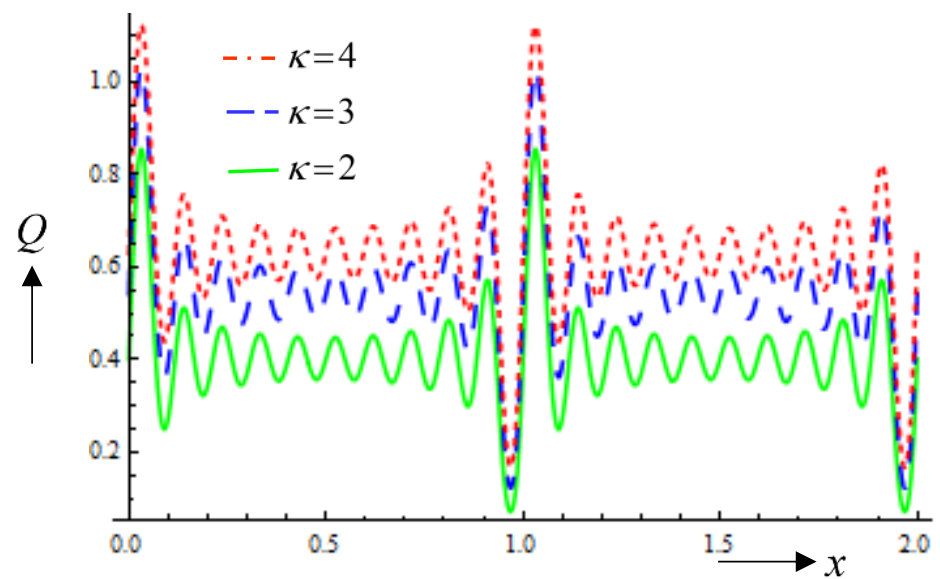

Fig.7. Effect of Debye length on volumetric flow rate with $h_{p l}=0.1, u_{e}=1$.

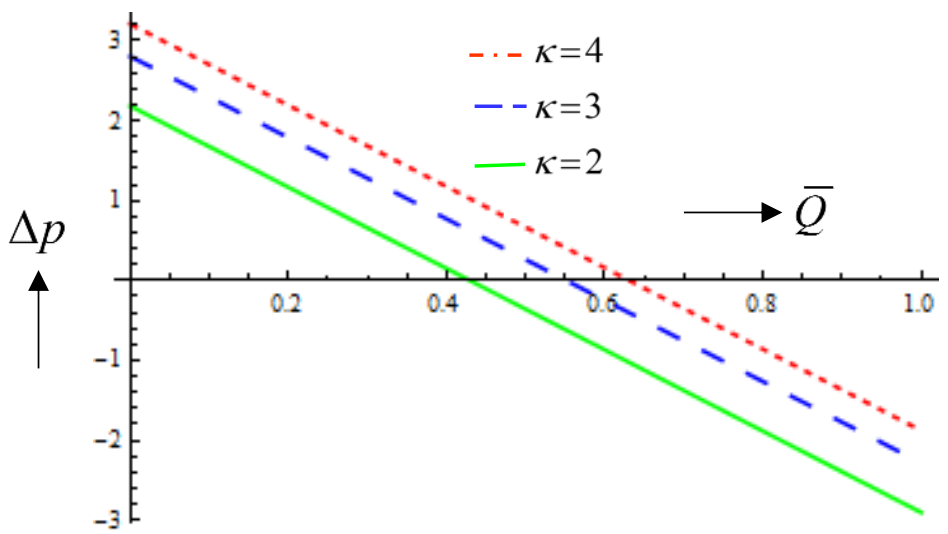

Fig.8. Effect of Debye length on pressure rise with $h_{p l}=0.1, u_{e}=1$.

(a)

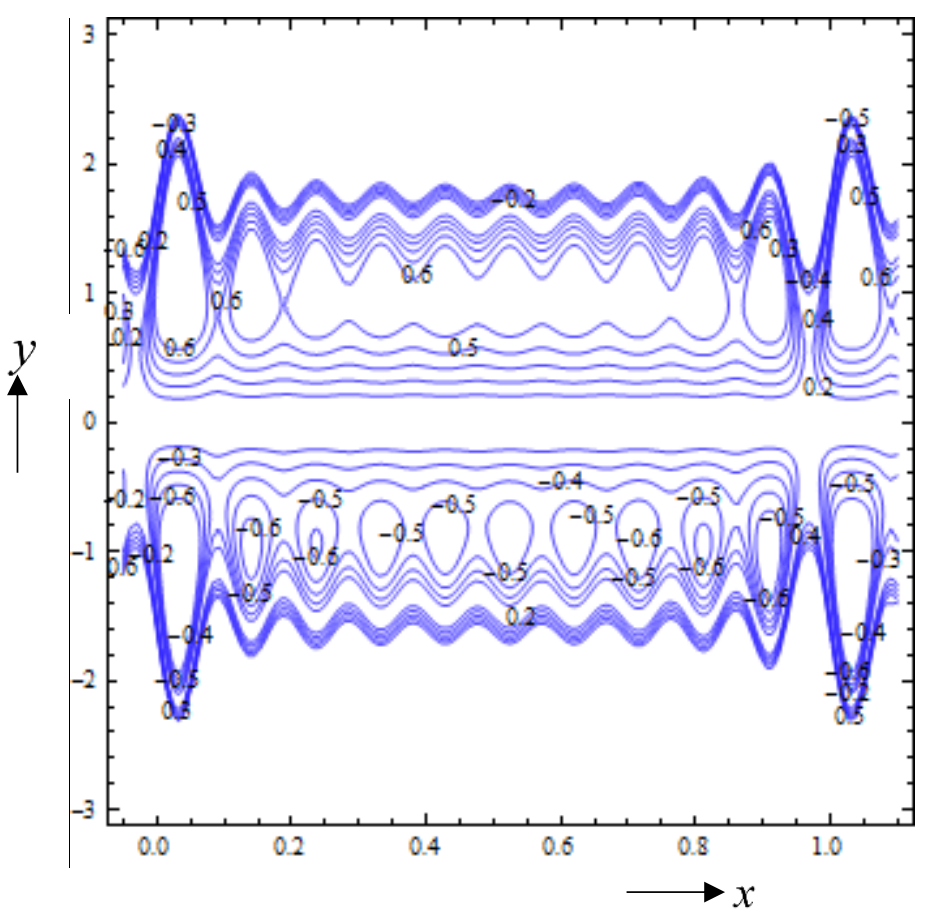



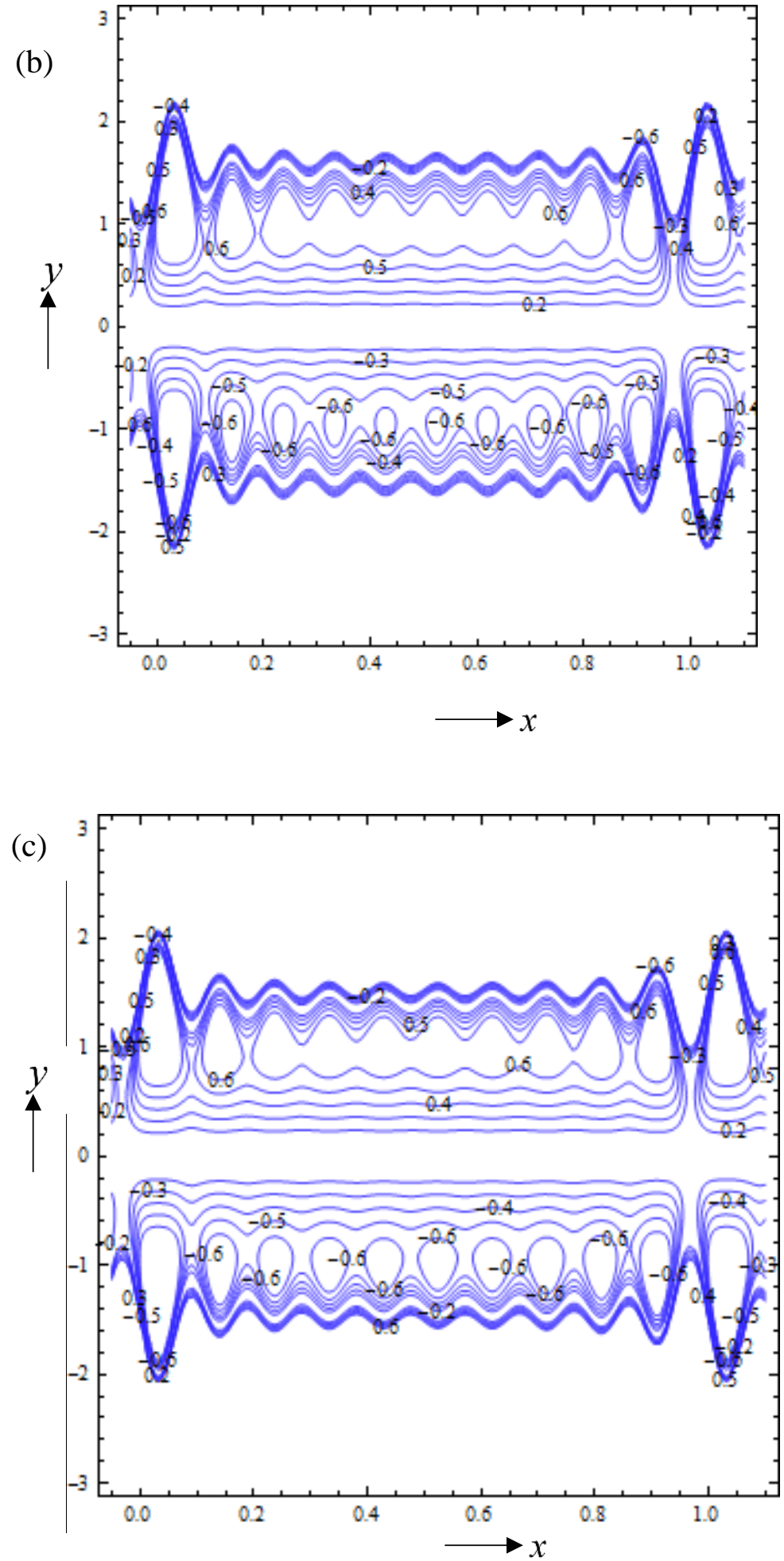

Fig.9. Stream lines for $\bar{Q}=0.6, h_{p l}=0.1, u_{e}=1$ with (a) $\kappa=2$ (b) $\kappa=3$ (c) $\kappa=4$. 


\subsection{Effects of Helmholtz-Smoluchowski velocity}

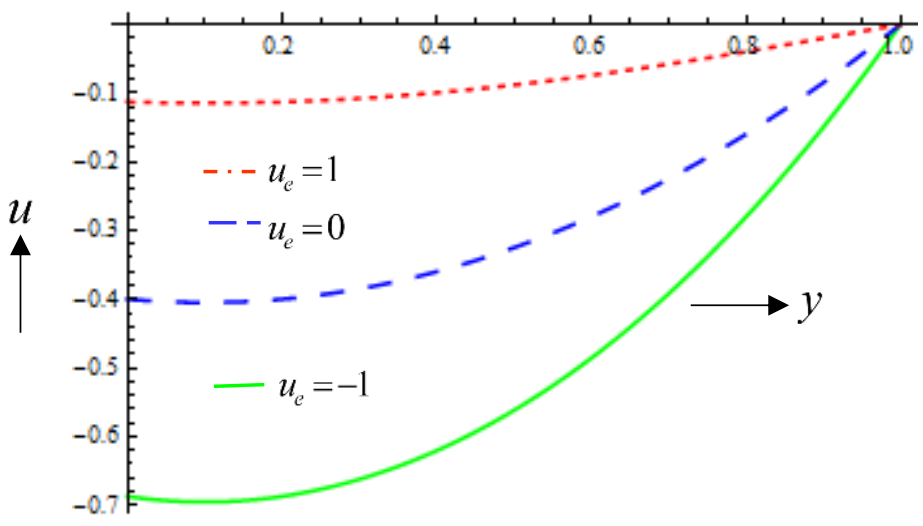

Fig.10. Effect of Helmholtz-Smoluchowski velocity on axial velocity with $x=1, \kappa=1$, $h_{p l}=0.1$.

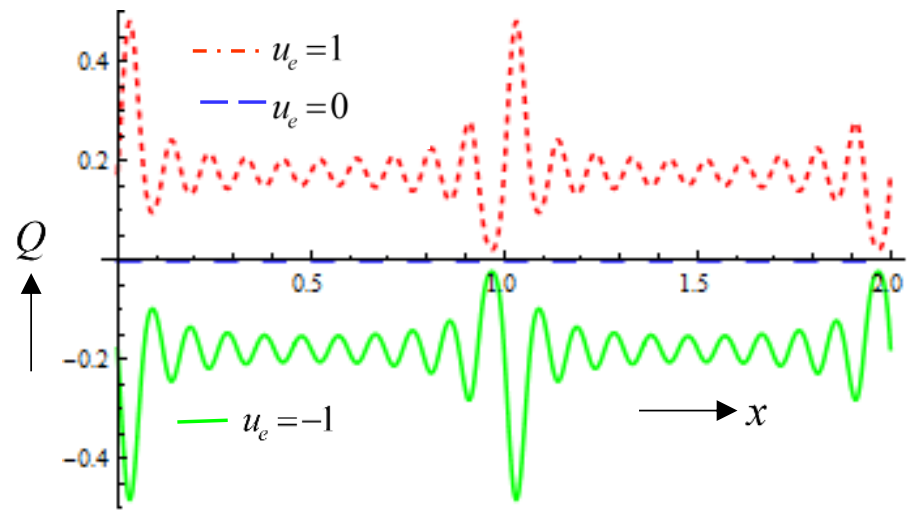

Fig.11. Effect of Helmholtz-Smoluchowski velocity on volumetric flow rate with $\kappa=1, h_{p l}=0.1$.

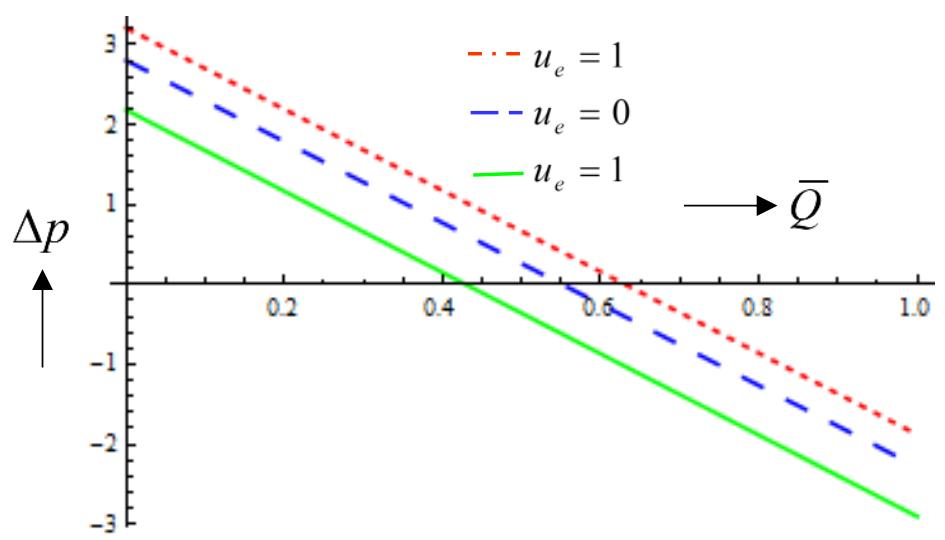

Fig.12. Effect of Helmholtz-Smoluchowski velocity on pressure rise with $\kappa=1, h_{p l}=0.1$. 
(a)

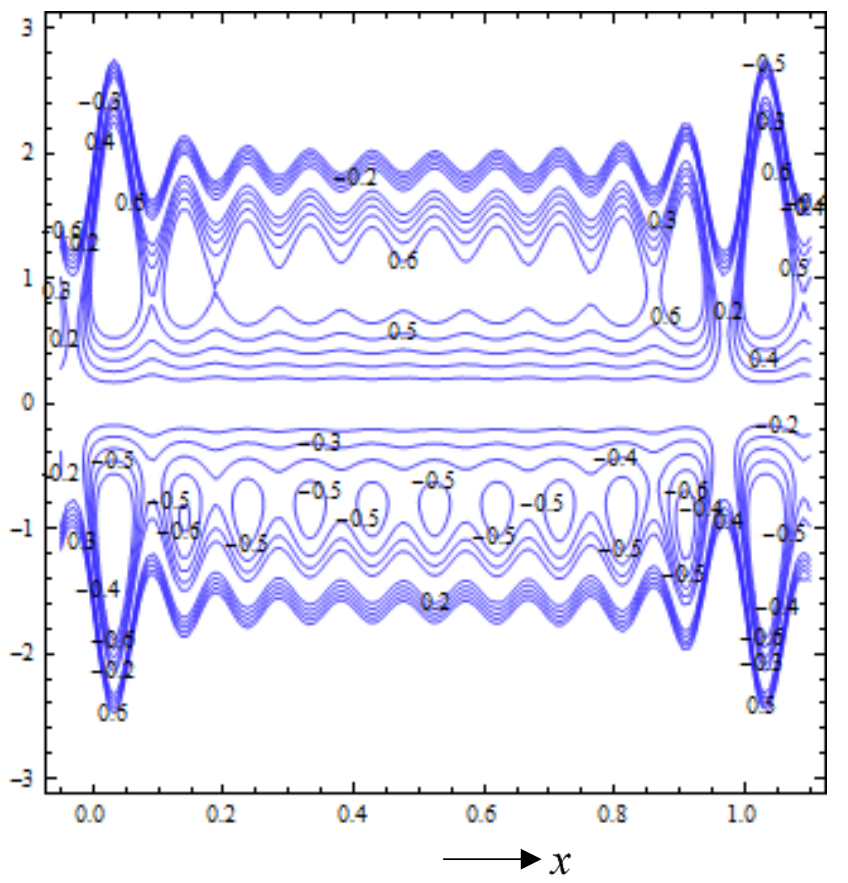

(b)

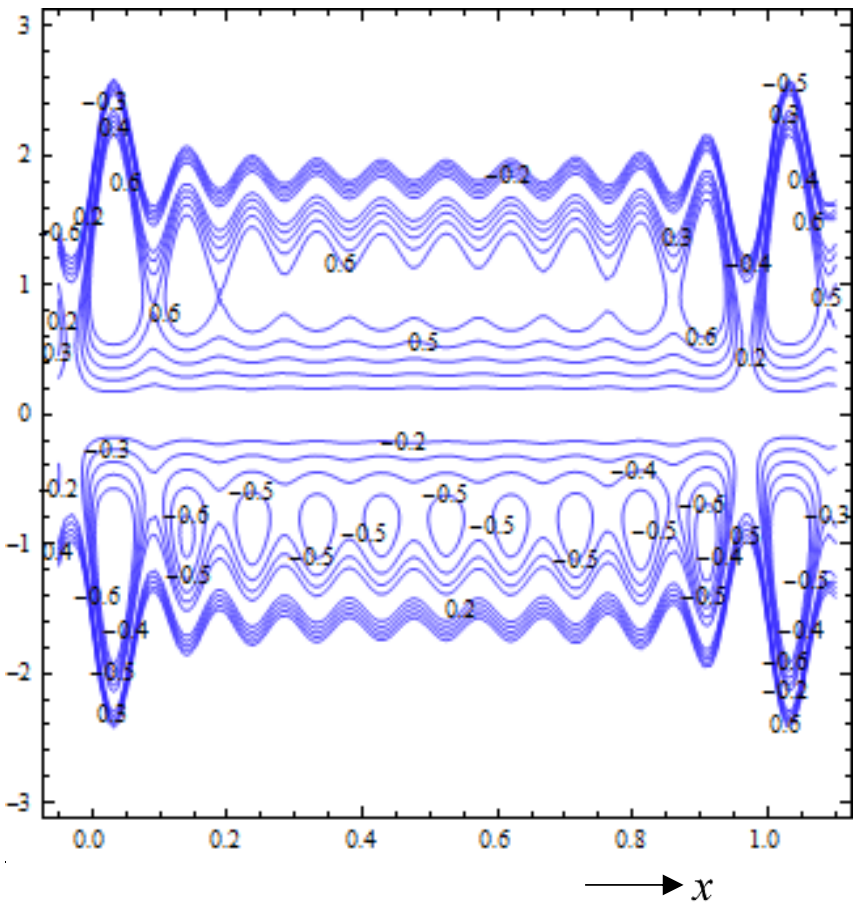




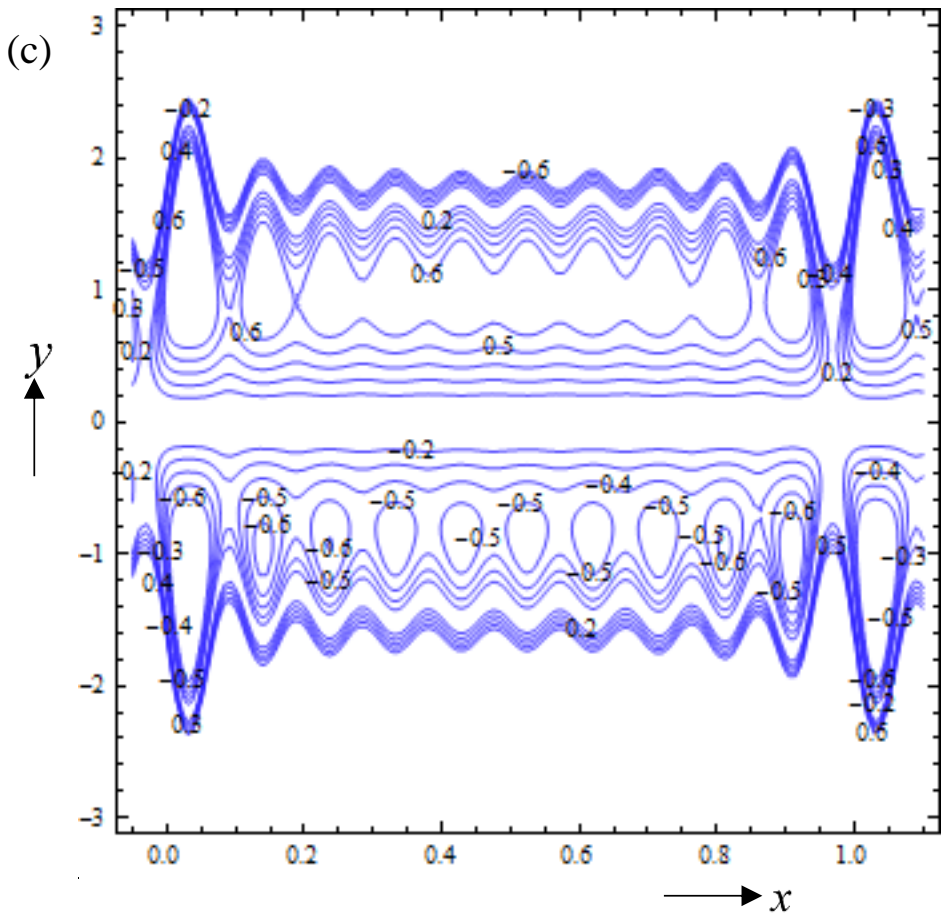

Fig.13. Stream lines for $\bar{Q}=0.6, \kappa=1, h_{p l}=0.1$ with (a) $u_{e}=0$ (b) $u_{e}=2$ (c) $u_{e}=5$.

Fig. 2 illustrates the evolution in axial velocity across the channel semi-span with variation in plug flow width $\left(h_{p l}\right)$. Due to symmetry only half the channel span is considered. Maximum velocity as expected arises at $y=0$ (channel centre line) with vanishing of velocity at the microchannel boundary $(y=h)$. The plug flow width corresponds to the cross-channel core region width. When this is increased the viscoplastic fluid is permitted to flow with more ease. The axial flow is therefore boosted. When the plug flow width is zero the core region has not developed and significant retardation to the axial flow is induced. The Bingham viscoplastic fluid behaves as a solid when the applied shear stress is less than the yield stress. However once the yield stress is exceeded, as in the present simulations, the viscoplastic fluid flows like a conventional fluid. Bingham plastics are a special class of viscoplastic fluids that exhibit a linear behavior of shear stress against shear rate. Although this model is simple it does represent quite accurately reported behaviour in electrokinetic microfluides systems, as elaborated by Tang et al. [42]. The Bingham plastic also exhibits a smooth velocity profile (parabolic across the whole channel). There is no alternation in the magnitudes of velocity indicating that the flow remains orientated in one direction.

Fig. 3 depicts the evolution in volumetric flow rate with axial distance $(x)$ for various plug flow width values. In all plots the multiple wave amplitudes of the peristaltic waves are clearly 
captured. Markedly higher values of volumetric flow rate arise at the entry zone to the microchannel $(x=0)$, again mid-way along the channel but are significantly reduced with further increase in axial distance plummeting at the exit zone $(x \sim 1.0)$. With increasing plug flow width, as anticipated, the flow is accelerated and volumetric flow rate is enhanced considerably. The nature of plug flow width is intimately associated with the type of Bingham plastic, as noted by Nguyen and Boger [43]. Judicious selection of the appropriate working fluid therefore ensures that while yield stress behaviour is still present, it does not inhibit plug flow growth.

Fig. 4 illustrates the evolution in pressure difference across one wavelength $(\Delta p)$ with time averaged volumetric flow rate $(\bar{Q})$ for selected plug width flow values. Three pumping regimes, as first identified by Shapiro et al. [27] are significant in peristaltic propulsion. These are the pumping region $(\Delta p>0)$, the augmented pumping region $(\Delta p<0)$, and the free pumping region $(\Delta p=0)$. Evidently in the pumping region, pressure difference decreases with flow rates. The $\Delta p$ - $\bar{Q}$ relationship is clearly an inverse linear relationship i.e. pressure difference decreases with increasing time averaged flow rate. However the relationship between pressure difference and flow rate strongly depends on whether pressure difference is negative or positive. When plug flow width is zero and pressure difference is positive, lower pressure difference is computed compared with non-zero plug width. However the dominant trend (when pressure difference is negative) is a decrease in pressure difference with increasing plug flow width which is accentuated with greater averaged volumetric flow rate. The maximum deviation in profiles therefore corresponds to the maximum flow rate case.

Figs. 5 (a)-(c) visualize the streamline distributions for variation in plug flow width $\left(h_{p l}\right)$ with all other parameters constrained (Debye-Hückel parameter $(\kappa)$ i.e. Debye electroosmotic length $=$ Helmholtz-Smoluchowski velocity $\left(u_{e}\right)=$ unity). The entire micro-channel space is considered. These figures visualize a key characteristic of peristaltic flows, namely trapping phenomenon which relates to the stream lines circulation and formation of a trapped bolus. It allows the determination of reflux characteristics and also vortex growth and circulation intensity in peristaltic flows. Two sets of trapping zones are present - one above the centreline and one below. These zones contain large boluses at the entry and exit zones which progressively shrink in the intermediate zone. Multiple small boluses exist for $h_{p l}=0$ in both sets below and above the channel centre line. With an increase in plug flow width the upper 
row of boluses is eliminated and the lower row contracts significantly. Trapping is therefore largely suppressed with increasing plug flow width i.e. variation in Bingham viscoelasticity.

Figs. 6- 9(a)-(c) depict the impact of the electrical double layer thickness i.e. Debye-length parameter $(\kappa)$ on respectively the axial flow velocity, volumetric flow rate, pressure difference and streamline distributions. Since the induced electrical field is dwarfed by the externally applied electric field, streaming potential is discarded in the electroosmotic flow. When the ions move in the diffuse layer, they pull the liquid along with them in the opposite direction to the pressure driven flow. This effect is inversely proportional to the thickness of the electrical double layer. Hence as $\kappa$ values increase the migration of ions is inhibited and the axial flow is accelerated, as observed in fig. 6 . There will be a corresponding boost therefore in volumetric flow rates which will also be elevated with increasing electrical double layer thickness, as illustrated in fig. 7. Enhanced pumping is therefore achieved with larger $\kappa$ values. Distinct from the $\Delta p-\bar{Q}$ profiles computed in fig. 4, a consistent response is observed for all three pumping zones in fig. 8. In the the pumping region $(\Delta p>0)$ and the free pumping region $(\Delta p=0)$, an increase in electrical double layer thickness i.e. Debye-length parameter $(\kappa)$ significantly elevates pressure differences with increasing averaged volumetric flow rate. This trend is also sustained for the augmented pumping region $(\Delta p<0)$. Enhanced efficiency of peristaltic pumping is therefore attained with greater Debye-length parameter irrespective of the pumping zone considered. Figs 9a-c demonstrate that with an initial increase in Debye-length parameter $(\kappa)$ the lower row of boluses in the micro-channel is initially reduced in size; however with subsequent increase the boluses begin to grow again. The upper row of boluses remains largely unaffected and is not sensitive to changes in Debye-length parameter.

Finally figs. 10-13 present the response in axial flow velocity, volumetric flow rate, pressure difference and streamline distributions.to a change in the Helmholtz-Smoluchowski velocity $\left(u_{e}\right)$. The definition of this parameter is negative i.e. $u_{e}=-\frac{E_{x} \varepsilon \zeta}{\mu c}$. It is directly proportional to the strength of axial electrical field. For negative values of $u_{e}$, the electrical field acts in the positive axial direction. It therefore impedes the axial flow (fig. 10) and this decreases axial velocity. However for positive values of $u_{e}$, the axial field is orientated in the reverse $x$-direction and this serves to accelerate the axial flow since the electroosmotic body forces assists momentum diffusion in this scenario. The direction of axial field therefore has a profound 
effect on flow characteristics. Volumetric flow rate (fig. 11) is therefore also boosted for positive values of $u_{e}$, and reduced for negative values of $u_{e}$. The oscillatory nature of the peristaltic flow is evident from the volumetric flow rate plot and shows peaks again at the entry and intermediate zones of the micro-channel length with a reduction at the exit zone. Pressure difference is found to decrease with time-averaged volumetric flow rate (fig. 12) and is always lower for negative values of $u_{e}$ (aligned electrical axial field) whereas it is higher for positive values of $u_{e}$ (reverse electrical axial field), for all three (free, standard and augmented) pumping zones. Figs. 13a-c exhibit the modification in bolus dynamics with increasing positive values of Helmholtz-Smoluchowski velocity $\left(u_{e}\right)$. It is apparent that no tangible alteration is induced in the upper row of boluses, whereas there is a notable growth in the lower row of boluses. An increase in positive Helmholtz-Smoluchowski velocity implies that the axial electrical field is progressively stronger in the reverse $x$-direction. This encourages trapping in the lower microchannel half space. Overall the presence of an electrokinetic body force effect induces nontrivial modifications in streamline distributions.

\section{Conclusions}

An analytical investigation of microvascular non-Newtonian blood flow model has been conducted. Multiple amplitudes of peristaltic waves have been considered and the Bingham viscoplastic rheological material model has been adopted. The non-dimensional boundary value problem has been solved with integration methods. Numerical evaluation of the closedform solutions has been performed with Mathematica symbolic software. The effects of Bingham plug flow width, Debye electrokinetic length and Helmholtz-Smoluchowski velocity (maximum electroosmotic velocity) on the axial velocity, averaged volumetric flow rate, pressure difference and stream function distribution have been plotted graphically. The computations have shown that:

- An increase in plug flow width increases axial velocity, reduces volumetric flow rate, elevates pressure difference (only in the pumping region) and suppresses bolus magnitudes

- Increasing electrical Debye length accelerates the axial flow, volumetric flow rate and also pressure rise (whether in the augmented, standard or free pumping region) and initially reduces bolus size (in the lower microchannel half-space) whereas with subsequent increase it increases bolus magnitudes. 
- With positive Helmholtz-Smoluchowski velocity (reverse axial electrical field) the axial flow is strongly accelerated and there is a significant enhancement in volumetric flow rate and pressure difference (for all pumping zones). The opposite trends are computed with negative Helmholtz-Smoluchowski velocity (aligned axial electrical field.

- Furthermore there is a substantial amplification in size of the lower row boluses with increasing positive Helmholtz-Smoluchowski velocity whereas the upper row of boluses is unaltered.

\section{REFERENCES}

1. Diez, F.J.; Hernaiz, G.; Miranda, J.J.; Sureda, M., On the capabilities of nano electrokinetic thrusters for space propulsion, Acta Astronautica, 83, 97-107 (2013).

2. Y. Uematsu, Nonlinear electro-osmosis of dilute non-adsorbing polymer solutions with low ionic strength, Soft Matter, 11(37):7402-11. (2015).

3. N. A Mishchuk, Electro-osmosis of the second kind near the heterogeneous ionexchange membrane, Colloids and Surfaces A: Physicochemical and Engineering Aspects, 140(1):75-89 (1998).

4. Rafael Taboryski et al., Electro-osmotic flow micro pumps for cell positioning in biochips, Frontiers in Biochip Technology, 144-153 (2006).

5. Y.Gao, Cheng Wang, Teck Neng Wong, Chun Yang, Nam-Trung Nguyen and Kim Tiow Ooi, Electro-osmotic control of the interface position of two-liquid flow through a microchannel, J. Micromech. Microeng. 17, 358 (2007).

6. I. Gajda, I., Greenman, J., Melhuish, C., Santoro, C., Li BaiKun, Cristiani, P. and Ieropoulos, I., Electro-osmotic-based catholyte production by Microbial Fuel Cells for carbon capture, Water Research (Oxford),86, 108-115 (2015).

7. Neville C. Lockhart. Electro-osmotic dewatering of coal froth flotation concentrates, Fuel, 61, 780-781 (1982).

8. V. F. Hock, O. Marshall, M. McInerney, S. Morefield, Electro-osmotic pulse technology for corrosion prevention and control of water intrusion in below grade concrete structures, Advanced Materials Research, 38, 79-87 (2008).

9. G. Hwang et al., Electro-osmotic propulsion of helical nanobelt swimmers, The International Journal of Robotics Research, 30, 806-819 (2011). 
10. S. Liu, Q. Pu and J.J. Lu, Electric field-decoupled electroosmotic pump for microfluidic devices, J. Chromatography A, 1013 (2003) 57-64.

11. C.Y. Soong and S.H. Wang, Analysis of rotation-driven electrokinetic flow in microscale gap regions of rotating disk systems, Journal of Colloid and Interface Science, 269, 484-498 (2004).

12. F. Bianchi, R. Ferrigno, and H. H. Girault, Finite element simulation of an electroosmotic-driven flow division at a T-junction of microscale dimensions, Anal. Chem., 72, 1987-1993 (2000).

13. M. Wang, N. Pan, J. Wang and S. Chen, Lattice Poisson-Boltzmann simulations of electroosmotic flows in charged anisotropic porous media, Communications in Computational Physics, 2, 1055-1070 (2000).

14. C. Qi and C-O Ng., Electro-osmotic flow of a power-law fluid in a slit microchannel with gradually varying channel height and wall potential, European Journal of Mechanics - B/Fluids, 52, 160-168 (2015).

15. C.C. Cho, Chen, CL. and Chen, CK. Flow characteristics and mixing performance of electrokinetically driven non-Newtonian fluid in contraction-expansion microchannel, Rheol Acta., 51: 925-935 (2012).

16. Y. Huang, Juzheng Chen, Teck Neng Wong and Jong-Leng Liow,_Experimental and theoretical investigations of non-Newtonian electro-osmotic driven flow in rectangular microchannels, Soft Matter, 12, 6206-6213 (2016).

17. E. Jiménez et al., Start-up electroosmotic flow of Maxwell fluids in a rectangular microchannel with high zeta potentials, Journal of Non-Newtonian Fluid Mechanics, 227, 17-29 (2016).

18. P Abhimanyu et al., Transiences in rotational electro-hydrodynamics microflows of a viscoelastic fluid under electrical double layer phenomena, Journal of Non-Newtonian Fluid Mechanics, 231, 56-67 (2016).

19. C-O. $\mathrm{Ng}$, Combined pressure-driven and electro-osmotic flow of Casson fluid through a slit microchannel, Journal of Non-Newtonian Fluid Mechanics, 198, 1-9 (2013).

20. J.L. Viota, de Vicente, J., Ramos-Tejada, M.M. et al., Electrical double layer and rheological properties of yttria-stabilized zirconia suspensions in solutions of high molecular weight polyacrylic acid polymers, Rheologica Acta, 43, 645-656 (2004).

21. Hsin-Fu Huang,Cheng-Hung Yao, Electrokinetic diffusio-osmosis of viscoelastic Phan-Thien-Tanner liquids in slit microchannels, Journal of Non-Newtonian Fluid Mechanics, 206, 1-10 (2014). 
22. P. K. Swamee et al., Explicit equations for laminar flow of Bingham plastic fluids, Journal of Petroleum Science and Engineering, 76, 178-184 (2011).

23. X. Huang and M.H. Garcia, Perturbation solution for Bingham-plastic mudflows, ASCE Journal of Hydraulic Engineering, 123, 10-35 (1997).

24. M. Danish, S. Kumar, S. Kumar, Approximate explicit analytical expressions of friction factor for flow of Bingham fluids in smooth pipes using Adomian decomposition method, Communications in Nonlinear Science and Numerical Simulation, 16, 239-251 (2011).

25. M-R. Mohammadi and A. J. Moghadam, Heat transfer and entropy generation analysis of Bingham plastic fluids in circular microchannels, ASME J. Thermal Sci. Eng. Appl., 7(4), 041019 (2015) (11 pages).

26. Y.C. Fung, Biomechanics, Springer, New York (1990).

27. A. H. Shapiro, M. Y. Jaffrin and S. L. Weinberg, Peristaltic pumping with long wavelengths at low Reynolds number, J. Fluid Mech. 37, 799-825 (1969).

28. S.W. Walker and M.J Shelley, Shape optimization of peristaltic pumping, Journal of Computational Physics, 229, 1260-1291 (2010).

29. M. R. Kaimal, Peristaltic pumping of a Newtonian fluid with particles suspended in it at low Reynolds number under long wavelength approximations, ASME J. Appl. Mech. 5(1):32-36 (1978).

30. D. Tang and M.C. Shen, Peristaltic transport of a heat-conducting fluid subject to Newton's cooling law at the boundary, International Journal of Engineering Science, 27, 809-825 (1989).

31. P. Nagarani and A. Lewis, Peristaltic flow of a Casson fluid in an annulus, Korea-Aust. Rheol. Journal, 24: 1-9 (2012).

32. D. Tripathi and O. Anwar Bég, Mathematica numerical simulation of peristaltic biophysical transport of a fractional viscoelastic fluid through an inclined cylindrical tube, Computer Methods in Biomechanics and Biomedical Engineering, 18, 1648-1657 (2015).

33. Y. Abd elmaboud, Kh.S. Mekheimer, Non-linear peristaltic transport of a second-order fluid through a porous medium, Applied Mathematical Modelling, 35, 2695-2710 (2011).

34. N.P. Khabazi et al., Peristaltic flow of Bingham fluids at large Reynolds numbers: A numerical study, Journal of Non-Newtonian Fluid Mechanics, 227, 30-44 (2016). 
35. D. Tripathi and O. Anwar Bég Mathematical modelling of peristaltic propulsion of viscoplastic fluids, Proc. IMECHE-Journal of Engineering in Medicine, 228 (1): 67-88 (2014).

36. S. Chakraborty, Augmentation of peristaltic microflows through electro-osmotic mechanisms, J. Phys. D: Appl. Phys. 39, 5356-5363 (2006).

37. D. Tripathi, A. Yadav and O. Anwar Bég, Analytical study of electro-osmosis modulated capillary peristaltic hemodynamics, J. Mechanics in Medicine and Biology, 17, No. 5 (2017) 1750052 (22 pages) DOI: 10.1142/S021951941750052X

38. P. Goswami et al., Electrokinetically modulated peristaltic transport of power-law fluids, Microvascular Research, 103, 41-54 (2016).

39. D. Tripathi, A. Yadav and O. Anwar Bég, Electro-kinetically driven peristaltic transport of viscoelastic physiological fluids through a finite length capillary: mathematical modelling, Mathematical Biosciences, 283, 155-168 (2017).

40. G. C. Shit et al., Electro-osmotic flow of power-law fluid and heat transfer in a microchannel with effects of Joule heating and thermal radiation, Physica A: Statistical Mechanics and its Applications, 462, 1040-1057 (2016).

41. D. Tripathi, A. Yadav and O. Anwar Bég, Electro-osmotic flow of couple stress fluids in a micro-channel propagated by peristalsis, European Physical Journal Plus, 132: 173-188 (2017).

42. G. Tang, Y. Lu, Y. S., Non-Newtonian flow in microchannels, International Journal of Modern Physics: Conference Series, 4, 1460385 (11 pages) (2014).

43. Q D Nguyen, and D V Boger, Measuring the flow properties of yield stress fluids, Annual Review of Fluid Mechanics, 24, 47-88 (1992). 